05

\title{
Моделирование реономного поведения сегнетоэлектроупругих материалов при циклическом нагружении
}

\author{
(C) А.C. Семенов, С.М. Лобанов ब \\ Санкт-Петербургский политехнический университет Петра Великого, \\ 195251 Санкт-Петербург, Россия \\ ฯ e-mail: slobanov92@yandex.ru
}

(Поступило в Редакцию 4 апреля 2017 г. В окончательной редакции 14 марта 2018 г.)

\begin{abstract}
Предложена феноменологическая модель нелинейного связанного электромеханического поведения поликристаллической сегнетопьезокерамики при сложном многоосном воздействии, учитывающая чувствительность к скорости нагружения и ориентированная на использование при решении краевых задач. В рамках единой модели рассмотрена возможность описания эффектов ползучести и чувствительности кривых гистерезиса к частоте внешнего воздействия. Произведено сравнение прогнозов модели с результатами экспериментов на макроскопических образцах из сегнетопьезокерамики PZT PIC 151 при циклическом нагружении. Сравнение результатов расчетов с прогнозами альтернативных моделей продемонстрировало более высокую точность предложенной модели.
\end{abstract}

DOI: $10.21883 / J T F .2018 .10 .46496 .2286$

\section{Введение}

Поликристаллическая сегнетопьезокерамика находит широкое применение на практике в качестве элементов топливных инжекторов, нанопозиционеров, микромоторов, гасителей вибраций, пьезотрансформаторов, приводов электронных микроскопов. Для корректной оценки точности позиционирования, прочности и долговечности вышеперечисленных устройств требуется учет нелинейного связанного электромеханического поведения, чувствительного к скорости нагружения. Это приводит к необходимости разработки и использования в расчетах моделей материала, учитывающих эффекты вязкости.

Экспериментальное изучение реономных эффектов сегнетоэлектриков/сегнетоэластиков (называемых далее сегнетоэлектроупругими материалами), начинающееся с прямого анализа времени переполяризации и динамики доменной структуры [1] и охватывающее исследования процессов неустановившейся ползучести деформаций [2-7] и поляризации [2-4,8] на макроуровне, релаксацию напряжений [9] и электрического поля [10], чувствительность кривых диэлектрического $[11,12]$ и электромеханического гистерезиса (в форме бабочки) $[11,12]$ к частоте внешнего воздействия, служит основой для разработки и верификации моделей материала. Трудности исследования неравновесных явлений в полидоменных сегнетоэлектроупругих материалах связаны с их неоднородностью вследствие наличия доменных стенок, обладающих множеством метастабильных состояний, отделенных друг от друга и от основного равновесного состояния большими энергетическими барьерами из-за наличия дефектов. В результате одновременно наблюдаются как быстрые процессы переполяризации, так и медленные термоактивационные процессы [10].

Первые феноменологические модели сегнетоэлектроупругого материала были предложены В.Л. Гинзбургом [13] и А.Ф. Девонширом [14]. В этих моделях, основанных на теории фазовых переходов Ландау, для свободной энергии используется полиномиальное представление с четными степенями параметра порядка, в качестве которого вводилась поляризация. Простейшая модель кинетики переключения определяется уравнением Ландау-Халатникова [15], описывающим пространственно однородное (бездоменное) переключение. Учет доменного механизма переключения произведен в модели Колмогорова-Аврами-Ишибаши [16-19] на основе использования статистической теории процессов зарождения и неограниченного роста доменов. Кинетика начальной стадии переключения одноосных сегнетоэлектриков-сегнетоэластиков в области слабой метастабильности описана в работе [20]. Релаксационная модель процессов переключения в кристаллических сегнетоэлектриках рассмотрена в работах [21,22].

При решении трехмерных связанных краевых задач для элементов конструкций с нетривиальной геометрией в условиях, близких к изотермическим при температуpax значительно ниже температуры Кюри, использование в расчетах микромеханических моделей материала, детально учитывающих кинетику доменной структуры, требует значительных вычислительных затрат и большого объема исходных экспериментальных данных. Поэтому наиболее рационально использование феноменологических (макроскопических) моделей сегнетоэлектроупругого материала, демонстрирующих экономичность процедур численного решения краевых задач [23] и обладающих простотой методов определения характеристик материала. Примеры подобных моделей рассматривались в [24-27]. Областью применения данного класса макроскопических моделей является анализ процессов деформирования, первичной поляризации, прочности, долговечности и трещиностойкости элементов перечисленных выше конструкций.

На основе использования принципа максимума диссипации при описании нелинейного склерономного 
поведения поликристаллической сегнетопьезокерамики, Ч. Ландисом предложена трехмерная связанная феноменологическая модель [26]. В настоящей работе рассматривается дальнейшее развитие этой модели в целях наиболее точного предсказания реономного поведения сегнетоэлектроупругого материала. Сравнение прогноза предложенной модели при постоянном уровне внешнего воздействия (ползучести) производилось в [28]. Целью выполненных исследований является сравнение поведения модели при циклическом нагружении с результатами экспериментов и с прогнозами других моделей, получивших наиболее широкое распространение на практике.

\section{1. Определяющие уравнения}

$\mathrm{B}$ соответствии с парадигмой стандартного материала с диссипацией [29] для задания определяющих уравнений (уравнений состояния) вводятся два потенциала: плотность свободной энергии (термодинамический потенциал Гельмгольца) $\Psi$ и потенциал диссипации $\Phi$. Зависимость от истории нагружения учитывается на основе введения двух внутренних переменных состояния: тензора остаточных деформаций $\varepsilon^{r}$ и вектора поляризации $\mathbf{P}^{r}$. Принимается представление свободной энергии, включающее обратимую и необратимую части [26]:

$$
\Psi=\Psi^{s}\left(\varepsilon, \mathbf{D}, \varepsilon^{r}, \mathbf{P}^{r}\right)+\Psi^{r}\left(\varepsilon^{r}, \mathbf{P}^{r}\right) .
$$

Использование концепции стандартного материала с диссипацией обеспечивает условия существования и единственности решения начально-краевой задачи.

В предположении линейного пьезоэлектрического отклика первое слагаемое $\Psi^{s}$ в выражении (1) определяется уравнением [26]:

$$
\begin{aligned}
& \Psi^{s}=\frac{1}{2}\left(\varepsilon-\varepsilon^{r}\right) \cdot{ }^{4} \mathbf{C}^{D} \cdot \cdot\left(\varepsilon-\varepsilon^{r}\right) \\
& -\left(\mathbf{D}-\mathbf{P}^{r}\right) \cdot{ }^{3} \mathbf{h} \cdot\left(\varepsilon-\varepsilon^{r}\right)+\frac{1}{2}\left(\mathbf{D}-\mathbf{P}^{r}\right) \cdot \boldsymbol{\beta}^{\varepsilon} \cdot\left(\mathbf{D}-\mathbf{P}^{r}\right),
\end{aligned}
$$

где $\varepsilon-$ тензор деформации, D - вектор электрического смещения, ${ }^{4} \mathbf{C}^{D}-$ тензор упругих модулей (четвертого ранга, обладает симметрией $C_{i j k l}^{D}=$ $\left.=C_{j i k l}^{D}=C_{i j l k}^{D}=C_{k l i j}^{D}\right), \boldsymbol{\beta}^{\varepsilon}-$ тензор диэлектрической проницаемости (второго ранга, обладает симметрией $\left.\beta_{i j}^{\varepsilon}=\beta_{j i}^{\varepsilon}\right),{ }^{3} \mathbf{h}-$ тензор пьезоэлектрических коэффициентов (третьего ранга, обладает симметрией $h_{i j k}=h_{i k j}$ ). Вышеперечисленные тензоры ${ }^{3} \mathbf{C}^{D}, \boldsymbol{\beta}^{\varepsilon},{ }^{3} \mathbf{h}$ допускают в общем случае зависимость от внутренних переменных.

Определяющие уравнения, получаемые из условия неотрицательности диссипации, позволяют определить на основе выражения обратимо запасенной свободной энергии $\Psi^{s}$ тензор напряжения $\boldsymbol{\sigma}$ и вектор напряженности электрического поля $\mathbf{E}[23]$ :

$$
\left\{\begin{array}{l}
\boldsymbol{\sigma}=\partial_{\varepsilon} \Psi={ }^{4} \mathbf{C}^{D} \cdots\left(\varepsilon-\varepsilon^{r}\right)-\left(\mathbf{D}-\mathbf{P}^{r}\right) \cdot{ }^{3} \mathbf{h}, \\
\mathbf{E}=\partial_{\mathbf{D}} \Psi=-{ }^{3} \mathbf{h} \cdot\left(\varepsilon-\varepsilon^{r}\right)+\boldsymbol{\beta}^{\varepsilon} \cdot\left(\mathbf{D}-\mathbf{P}^{r}\right) .
\end{array}\right.
$$

Необратимая часть свободной энергии $\Psi^{r}$ определяет форму гистерезисных кривых (упрочнение и насыщение) и допускает представление в виде аддитивного разложения на чисто электрическую $\Psi^{\varepsilon}$, чисто механическую и $\Psi^{m}$ и смешанную $\Psi^{e m}$ составляющие:

$$
\Psi^{r}\left(\varepsilon^{r}, \mathbf{P}^{r}\right)=\Psi^{e}\left(\mathbf{P}^{r}\right)+\Psi^{e m}\left(\varepsilon^{r}, \mathbf{P}^{r}\right)+\Psi^{m}\left(\varepsilon^{r}\right) .
$$

Степенные зависимости могут рассматриваться в качестве простейших аналитических аппроксимаций [26]:

$$
\Psi^{e}\left(\left|\mathbf{P}^{r}\right|\right)=\frac{H_{e} P_{0}^{2}}{m_{e}-1}\left[\frac{1}{m_{e}-2}\left(1-\frac{\left|\mathbf{P}^{r}\right|}{P_{0}}\right)^{2-m_{e}}-\frac{\left|\mathbf{P}^{r}\right|}{P_{0}}\right],
$$

где $H_{e}, P_{0}, m_{e}-$ константы материала, определяющие начальное упрочнение, поляризацию насыщения и показатель, характеризующий скорость насыщения. Выражения для $\Psi^{m}$ и $\Psi^{e m}$ вводятся аналогичным образом (см. [26]).

Обобщенные силы $\hat{\boldsymbol{\sigma}}$ и $\hat{\mathbf{E}}$, сопряженные с внутренними переменными $\varepsilon^{r}$ и $\mathbf{P}^{r}$, определяются равенствами

$$
\left\{\begin{array}{l}
\hat{\boldsymbol{\sigma}}=-\partial_{\varepsilon^{r}} \Psi=\boldsymbol{\sigma}-\partial_{\varepsilon^{r}} \Psi^{r}=\boldsymbol{\sigma}-\boldsymbol{\sigma}_{B}, \\
\hat{\mathbf{E}}=-\partial_{\mathbf{P}^{r}} \Psi=\mathbf{E}-\partial_{\mathbf{P}^{r}} \Psi^{r}=\mathbf{E}-\mathbf{E}_{B},
\end{array}\right.
$$

где $\boldsymbol{\sigma}_{B}=\partial_{\varepsilon^{r}} \Psi^{r}$ и $\mathbf{E}_{B}=\partial_{\mathbf{P}^{r}} \Psi^{r}-$ тензор микронапряжений и вектор остаточного электрического поля, характеризующие кинематическое упрочнение.

Диссипативное неравенство с использованием обобщенных сил допускает представление

$$
\delta=\boldsymbol{\sigma} \cdot \dot{\varepsilon}+\mathbf{E} \cdot \dot{\mathbf{D}}-\dot{\Psi}=\hat{\boldsymbol{\sigma}} \cdot \dot{\varepsilon}^{r}+\hat{\mathbf{E}} \cdot \dot{\mathbf{P}}^{r} \geq 0 .
$$

В пространстве обобщенных сил задается диссипативный потенциал (второй базовый потенциал, потенциал ползучести), определяемый выражением

$$
\Phi=A\left(\frac{\hat{\mathbf{E}} \cdot \hat{\mathbf{E}}}{E_{H}^{2}}+\frac{3}{2} \frac{\hat{\mathbf{s}} \cdot \hat{\mathbf{s}}}{\sigma_{H}^{2}}+\beta \frac{\hat{\mathbf{E}} \cdot \hat{\mathbf{s}} \cdot \hat{\mathbf{s}} \cdot \hat{\mathbf{E}}}{E_{H}^{2} \sigma_{H}^{2}}\right)^{m_{v}},
$$

где $\hat{\boldsymbol{s}}=\operatorname{dev} \hat{\boldsymbol{\sigma}}=\hat{\boldsymbol{\sigma}}-1$ tr $\hat{\boldsymbol{\sigma}}-$ девиатор активных напряжений, $A$ и $m_{v}-$ константы материала, характеризующие вязкие свойства, $E_{H}$ и $\sigma_{H}-$ скалярнозначные функции внутренних переменных, позволяющие учесть изотропное упрочнение. Введение последних принципиально для корректного описания процессов неустановившейся ползучести [28]. Безразмерный параметр $\beta$ определяет эффекты перекрестной электромеханической связанности. При ограничении рассмотрением чисто механического поведения выражение в правой части (8) сводится к интенсивности напряжений по Мизесу.

Для внутренних переменных (тензора остаточных деформаций $\varepsilon^{r}$ и вектора спонтанной поляризации $\mathbf{P}^{r}$ ) вводятся уравнения эволюции, построенные по аналогии с нелинейным вязким телом:

$$
\left\{\begin{array}{l}
\dot{\varepsilon}^{r}=\partial_{\hat{\boldsymbol{\sigma}}} \Phi \\
\dot{\mathbf{P}}^{r}=\partial_{\hat{\mathbf{E}}} \Phi
\end{array}\right.
$$


Выбор уравнений эволюции в виде (9) позволяет априори удовлетворить условию неотрицательности диссипации (7) для выпуклой функции $\Phi(\hat{\boldsymbol{\sigma}}, \hat{\mathbf{E}})$. Условия выпуклости выполняются при изменении параметра $\beta$ в диапазоне $0 \leq \beta \leq 3$.

Входящие в $(8)$ функции $E_{H}$ и $\sigma_{H}$ могут быть выражены через функции упрочнения при ползучести $h_{e}$ и $h_{m}$ :

$$
\left\{\begin{array}{l}
E_{H}\left(\left|\mathbf{P}^{r}\right|\right)=E_{c} h_{e}^{-1 / 2 m_{v}}\left(\left|\mathbf{P}^{r}\right|\right), \\
\sigma_{H}\left(\left\|\varepsilon^{r}\right\|\right)=\sigma_{c} h_{m}^{-1 / 2 m_{v}}\left(\left\|\varepsilon^{r}\right\|\right),
\end{array}\right.
$$

где $E_{c}$ и $\sigma_{c}-$ коэрцитивное электрическое поле и напряжение. При исключении возможности описания изотропного упрочнения принимается $E_{H}=E_{c}, \sigma_{H}=\sigma_{c}$.

При описании процессов ползучести функции $h_{e}$ и $h_{m}$ оказывают непосредственное влияние на вид кривых ползучести и позволяют описать неустановившуюся стадию ползучести. Это может быть показано, например, при рассмотрении одноосного воздействия вдоль оси $\mathbf{e}_{3}$ $\left(\mathbf{E}=\mathbf{e}_{e} E_{e}\right.$ или $\left.\boldsymbol{\sigma}=\mathbf{e}_{3} \mathbf{e}_{3} \sigma_{33}\right)$, для которого следствием уравнений эволюции (9) с учетом (8) и (10) получаются соотношения:

$$
\begin{aligned}
& \dot{P}_{3}^{r}=\frac{2 m_{v} A}{E_{c}}\left(\frac{E_{3}-E_{B_{3}}}{E_{c}}\right)^{2 m_{v}-1} h_{e}\left(P_{3}^{r}\right), \\
& \dot{\varepsilon}_{33}^{r}=\frac{2 m_{v} A}{\sigma_{c}}\left(\frac{\sigma_{33}-\sigma_{B_{33}}}{\sigma_{c}}\right)^{2 m_{v}-1} h_{m}\left(\varepsilon_{33}^{r}\right) .
\end{aligned}
$$

Размерность констант $\dot{P}_{0}^{r}=2 m_{v} A / E_{c}$ и $\dot{\varepsilon}_{0}^{r}=2 m_{v} A / \sigma_{c}$, входящих в (11), совпадает с размерностью величин, стоящих в левых частях введенных равенств. Величины, стоящие в круглых скобках, и последние сомножители $h_{e}$ и $h_{m}$ являются безразмерными.

Ниже будет проанализирован ряд моделей сегнетоэлектроупругого материала с позиции возможности их представления в рамках рассмотренного формализма вычисления внутренних переменных (8)-(10) и предлагаемого этими моделями варианта вычисления функции упрочнения. При этом для простоты ограничимся случаем чисто электрического нагружения.

В исходной модели Ландиса 2002 г. [26] изотропное упрочнение не учитывается

$$
h_{e}^{L} \equiv 1
$$

Модель Лиу-Хубера 2006 г. [8] может рассматриваться как частный случай в рамках используемого подхода (8)-(10) при

$$
\begin{gathered}
h_{e}^{L H}=\frac{\exp \left(-g\left(\left|\mathbf{P}^{r}\right|\right)\right)}{t_{0} g^{\prime}\left(\left|\mathbf{P}^{r}\right|\right)}, \\
g\left(\left|\mathbf{P}^{r}\right|\right)=\frac{P_{s}}{P_{s}-\left|\mathbf{P}^{r}\right|}-\frac{P_{s}}{P^{*}+\left|\mathbf{P}^{r}\right|},
\end{gathered}
$$

где $P^{*}$ и $P_{s}-$ константы материала, характеризующие начальную и предельно допустимую поляризацию.
Функция упрочнения $h_{e}^{L H}$ вводится как функция абсолютного значения вектора поляризации. В данной модели предполагается отсутствие кинематического упрочнения $\mathbf{E}_{B}=0$. Механическое поведение в модели Лиу-Хубера не рассматривалось.

В модели Белова-Креера 2009 г. [30] функция упрочнения определяется в пространстве обобщенных сил следующим образом:

$$
\begin{aligned}
h_{e}^{B K}= & \frac{1}{E_{2}-E_{1}} \frac{E_{*}}{m} \exp \left(-m\left[1-\frac{|\hat{\mathbf{E}}|-E_{1}}{E_{*}}\right]\right) \\
& \times \begin{cases}1-\exp \left(-m \frac{|\hat{\mathbf{E}}|-E_{1}}{E_{*}}\right), & E_{1}<|\hat{\mathbf{E}}|<E_{2}, \\
1-\exp \left(-m \frac{E_{2}-E_{1}}{E_{*}}\right), & E_{2}<|\hat{\mathbf{E}}|,\end{cases}
\end{aligned}
$$

где $E_{1}, E_{2}, E_{*}, m-$ константы материала. В модели произведен учет кинематического упрочнения.

В настоящей работе вводится степенная функция упрочнения $h_{e}$, как упрощение функций, предложенных Лиу-Хубером (13) и Беловым-Креером (15):

$$
h_{e}=\left(\frac{\left|\mathbf{P}^{r}\right|}{P_{0}}\right)^{n}\left(1-\frac{\left|\mathbf{P}^{r}\right|}{P_{0}}\right)^{k},
$$

где $P_{0}$ - предельная поляризация при насыщении, $n$ и $k$ - константы материала. Первый сомножитель в правой части (16) характеризует упрочнение и позволяет описать неустановившуюся стадию ползучести (вводится по аналогии с моделями ползучести металлов), а второй сомножитель необходим для описания насыщения (при стремлении $\left|\mathbf{P}^{r}\right|$ к $P_{0}$ функция $h_{e}$ стремиться к нулю, блокируя дальнейшее изменение $\mathbf{P}^{r}$ ).

В модели Ландау-Гинзбурга-Халатникова в качестве необратимой части свободной энергии вместо (5) используется полиномиальное представление:

$$
\Psi^{e}=\frac{\alpha}{2}\left|\mathbf{P}^{r}\right|^{2}+\frac{\beta}{4}\left|\mathbf{P}^{r}\right|^{4}
$$

где $\alpha, \beta-$ константы материала. Уравнение Ландау-Халатникова [15] $\quad \xi \dot{\mathbf{P}}^{r}=-\partial_{\mathbf{p}^{r}} \Psi, \quad$ где $\xi-$ коэффициент демпфирования (феноменологический параметр, определяющий скорость переключения) приводит к уравнению эволюции $\mathbf{P}^{r}$ (для переходов первого рода):

$$
\xi \dot{\mathbf{P}}^{r}=\mathbf{E}-\alpha \mathbf{P}^{r}-\beta\left|\mathbf{P}^{r}\right|^{2} \mathbf{P}^{r} .
$$

Уравнение (17) также может быть получено на основе $(8)-(10)$ при $m_{v}=1 / 2, A=E_{c} / \xi$ и

$$
h_{e}^{L G H} \equiv 1 \text {. }
$$

Вектор остаточного электрического поля $\mathbf{E}_{B}=\partial_{\mathbf{P}} \Psi^{r}$ в этом случае вычисляется на основе выражения $\mathbf{E}_{B}=\alpha \mathbf{P}^{r}+\beta\left|\mathbf{P}^{r}\right|^{2} \mathbf{P}^{r}$. Следует отметить, что уравнение (17) описывает кинетику собственного переключения, не связанного с движением доменов. 
При описании кинетики переключения сегнетоэлектрических материалов (за исключением сверхтонких пленок) широкое распространение получила доменная теория Колмогорова-Аврами-Ишибаши [16-19]. В соответствии с данным подходом при одноосном циклическом нагружении $E=h(f t)$ на этапе нагрузки поляризация определяется равенством [19]

$$
P^{r}=P_{0}\left\{1-2 \exp \left[-f^{d} \Phi(E)\right]\right\},
$$

где $f$ - частота, $h$ - периодическая функция, $d-$ параметр, зависящий от формы растущих доменов и способа их зарождения

$\Phi(E)=\int_{0}^{E} C_{d}\left[\int_{E^{\prime}}^{E} v\left(E^{\prime \prime}\right)\left\{h^{\prime}\left(h^{-1}\left(E^{\prime \prime}\right)\right)\right\}^{-1} d E^{\prime \prime}\right]^{d} n_{E}\left(E^{\prime}\right) d E^{\prime}$,

где $v(E)$ - скорость движения доменной стенки, которая принимается пропорциональной внешнему электрическому полю с некоторым коэффициентом $a$, являющимся дополнительным параметром модели: $v(E)=a E$, $n_{E}(E)$ - количество зародышей на единицу объема, $C_{d}$ - константа, определяемая формой растущих доменов $\left(C_{d}=2, d=1\right.$ для доменов с параллельными доменными стенками). Зависимость объемной доли переключившихся доменов $q=\left(P^{r} / P_{0}+1\right) / 2$ от времени для стохастического процесса зародышеобразования определяется выражением [19]

$$
q=1-\exp \left[-t^{d+1} \Psi(E)\right],
$$

где $\Psi(E)=C_{d} v^{2}(E) n(E) /(d+1)$.

Соотношения, введенные выше для шести различных моделей для вычисления поляризации, могут быть обобщены и на случай вычисления тензора остаточной деформации. Однако на практике получила широкое распространение упрощенная квадратичная связь между деформациями и поляризаций [27]

$$
\varepsilon^{r}=\frac{3}{2} \frac{\varepsilon_{0}}{P_{0}^{2}} \operatorname{dev}\left(\mathbf{P}^{r} \mathbf{P}^{r}\right)=\frac{3}{2} \frac{\varepsilon_{0}}{P_{0}^{2}}\left(\mathbf{P}^{r} \mathbf{P}^{r}-\frac{1}{3} \mathbf{1} \mathbf{P}^{r} \cdot \mathbf{P}^{r}\right) .
$$

В одноосном случае соотношение (22) упрощается: $\varepsilon_{33}^{r}=\varepsilon_{0}\left(P_{3}^{r} / P_{0}\right)^{2}$.

\section{2. Сравнение результатов расчетов с экспериментальными данными}

При проведении расчетов использовалась программа CES (Constitutive Equation Etudio) [31], в рамках которой имплементированы все шесть вышеописанных моделей сегнетоэлектроупругого материала.

\section{1. Сравнение моделей при циклическом нагружении частотой $f=0.0025 \mathrm{~Hz}$}

Производится сравнение результатов расчетов с результатами экспериментов Жу [32] на изначально непо- ляризованных образцах поликристаллической сегнетопьезокерамики PZT PIC151 при переменном пилообразном электрическом поле с амплитудой $E=2 \mathrm{MV} / \mathrm{m}$, превышающей значение коэрцитивного поля $E_{c}=1 \mathrm{MV} / \mathrm{m}$, и частотой $f=0.0025 \mathrm{~Hz}$. Размер образцов составлял $5 \times 5 \times 15 \mathrm{~mm}^{3}$. Испытания проводились при комнатной температуре.

Результаты моделирования первого цикла нагружения с использованием моделей Ландау-Гинзбурга, Лиу-Хубера, Колмогорова-Аврами-Ишибаши и предложенной модели (16) с параметрами, указанными в табл. 1-4, представлены на рис. 1. Значение предельной остаточной деформации во всех моделях принималось равным $\varepsilon_{0}=3.3 \%$.

Из рис. 1, а видно, что при использовании модели Ландау-Гинзбурга удается получить близкие к экспериментальным данным значения основных параметров гистерезиса - предел остаточной поляризации и коэрцетивное поле, однако форма гистерезиса при расчете по этой модели заметно отличается от экспериментальной. Использование модели Лиу-Хубера позволяет улучшить точность по форме кривой нагрузки тем не менее при разгрузке наблюдаются значительные отличия. Схожая точность результатов наблюдается и при использовании моделей Ландиса и Белова-Креера. Модель Колмогорова-Аврами-Ишибаши позволяет повысить точность, но полного совпадения на всех участ-

Таблица 1. Параметры модели Ландау-Гинзбурга (17), (18) для PZT PIC151

\begin{tabular}{c|c|c|c|c}
\hline$E_{c}, \mathrm{MV} / \mathrm{m}$ & $\alpha, \mathrm{MV} \mathrm{m} / \mathrm{s}$ & $\beta, \mathrm{MV} \mathrm{m}^{5} / \mathrm{s}^{3}$ & $A, \mathrm{C} / \mathrm{m}^{2}$ & $m_{v}$ \\
\hline 1 & -2400000 & 60000000 & 30000 & 0.5
\end{tabular}

Таблица 2. Параметры модели Лиу-Хубера (13), (14) для PZT PIC151

\begin{tabular}{c|c|c|c|c|c|c}
\hline$E_{c}, \mathrm{MV} / \mathrm{m}$ & $P_{s}, \mathrm{C} / \mathrm{m}^{2}$ & $P^{*}, \mathrm{C} / \mathrm{m}^{2}$ & $H_{0}^{e}, \mathrm{~m} / \mathrm{F}$ & $m_{e}$ & $A, \mathrm{C} / \mathrm{m}^{2}$ & $m_{v}$ \\
\hline 1 & 0.39 & 0.01 & 400000 & 1.4 & $1.5 \cdot 10^{8}$ & 11.5
\end{tabular}

Таблица 3. Параметры модели Колмогорова-Аврами-Ишибаши (19), (20) для PZT PIC151

\begin{tabular}{c|c|c|c|c|c}
\hline$P_{0}, \mathrm{C} / \mathrm{m}^{2}$ & $a, \mathrm{~m}^{2} / \mathrm{s}^{2} / \mathrm{V}$ & $d$ & $C_{d}$ & $N_{0}$ & $n_{0}$ \\
\hline 0.37 & $2 \cdot 10^{-14}$ & 1 & 2 & 1 & 1.3
\end{tabular}

Таблица 4. Параметры предложенной модели (16) для PZT PIC151

\begin{tabular}{c|c|c|c|c|c|c|c}
\hline$E_{c}, \mathrm{MV} / \mathrm{m}$ & $P_{0}, \mathrm{C} / \mathrm{m}^{2}$ & $H_{0}^{e}, \mathrm{~m} / \mathrm{F}$ & $m_{e}$ & $A, \mathrm{C} / \mathrm{m}^{2}$ & $m_{v}$ & $n$ & $k$ \\
\hline 1 & 0.37 & 400000 & 1.4 & 1000 & 5.5 & -1.2 & -1.7
\end{tabular}



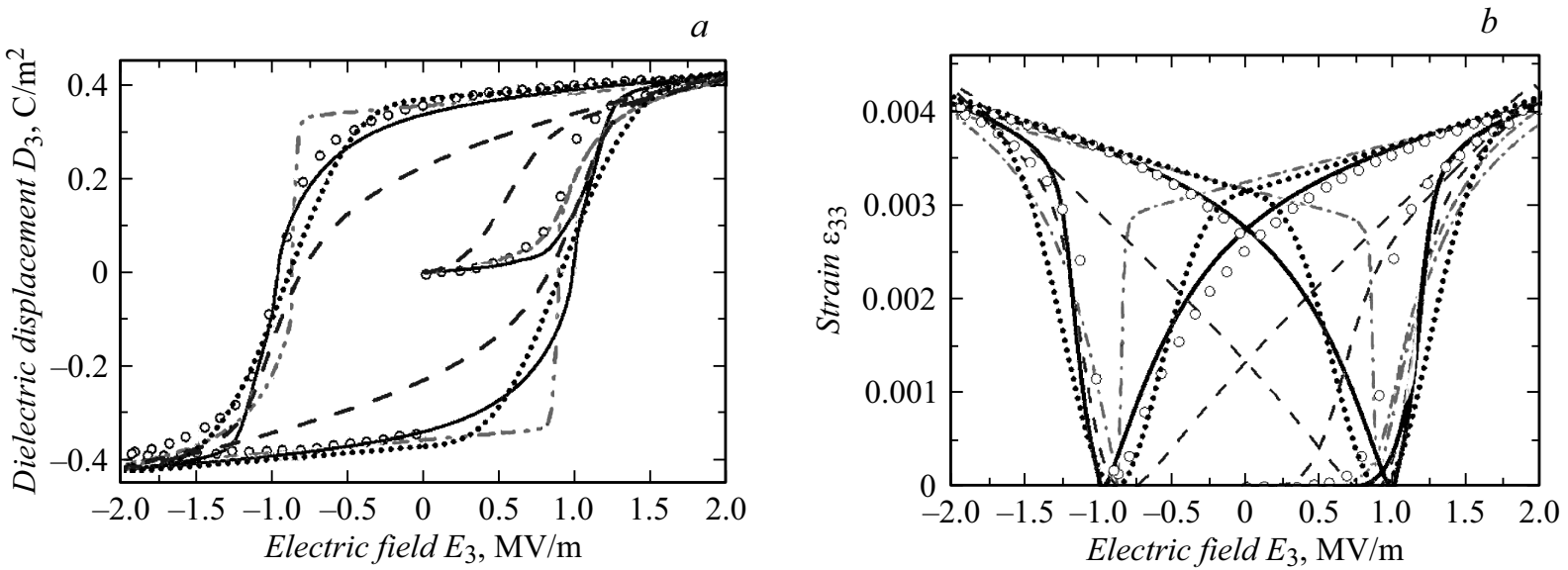

- Experiment

- - - Landau-Ginzbourg model

Liu-Huber model

….. KAI model

- Proposed model

Рис. 1. Сравнение результатов расчета с экспериментом при циклическом воздействии переменным электрическим полем $E=2 \mathrm{MV} / \mathrm{m}, f=0.0025 \mathrm{~Hz}: a-D-E$-гистерезис; $b-\varepsilon-E$-гистерезис.
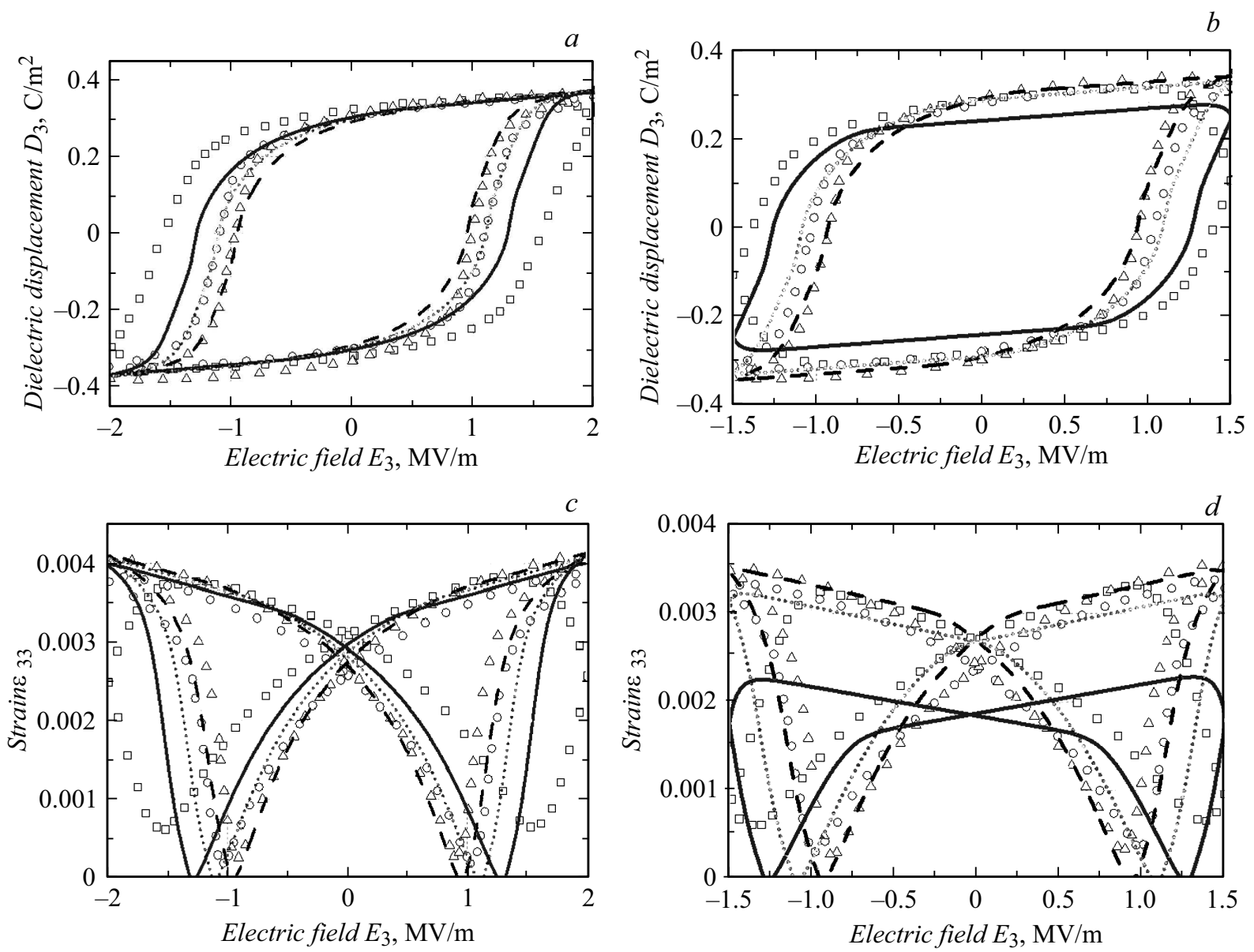

$\square \quad f=1 \mathrm{~Hz}$ Experimental

… $f=0.1 \mathrm{~Hz}$ Modeling

- $f=1 \mathrm{~Hz}$ Modeling

○ $f=0.1 \mathrm{~Hz}$ Experimental

$\triangle f=0.01 \mathrm{~Hz}$ Experimental

_ - $f=0.01 \mathrm{~Hz}$ Modeling

Рис. 2. Сравнение результатов расчета с экспериментом при циклическом воздействии переменным электрическим полем при различных частотах и амплитудах: $a-D-E$-гистерезис при $E_{a}=2 \mathrm{MV} / \mathrm{m} ; b-\varepsilon-E$-гистерезис при $E_{a}=2 \mathrm{MV} / \mathrm{m} ; c-D-E$ гистерезис при $E_{a}=1.5 \mathrm{MV} / \mathrm{m} ; d-\varepsilon-$ Е-гистерезис при $E_{a}=1.5 \mathrm{MV} / \mathrm{m}$. 
ках также не удается получить. Наилучшего совпадения результатов моделирования с экспериментальными данными удается достичь при использовании предложенной модели (16).

Аналогичные выводы могут быть сделаны также из анализа кривых электромеханического гистерезиса (в форме бабочки), показанных на рис. $1, b$.

\section{2. Сравнение в широком диапазоне изменения амплитуд и частот}

Результаты экспериментальных исследований влияния амплитуды и частоты нагружения на форму диэлектрического гистерезиса для образцов из сегнетоэлектрической керамики PZT PIC151 представлены в работе [33]. Рассматривались амплитуды $E=1,1.5,2 \mathrm{MV} / \mathrm{m}$ и частоты нагружения $f=0.01,0.1,1 \mathrm{~Hz}$.

В расчетах использовалась предложенная модель, определяемая уравнениями (16), (9), (8). Характеристики используемого материала практически совпадают с рассмотренными в предыдущем разделе (табл. 4). Однако вследствие различий в процессе начальной поляризации для данной серии образцов использовались значения $P_{0}=0.33 \mathrm{C} / \mathrm{m}, H_{0}^{e}=62000 \mathrm{~m} / \mathrm{F}, m=16$. Сравнение результатов численного моделирования с данными экспериментов при различных амплитудах и частотах представлено на рис. 2.

Анализ рис. 2 позволяет установить, что при относительно медленном нагружении (частоты порядка 0.01 и $0.1 \mathrm{~Hz}$ ) и амплитудах, превышающих коэрцетивное поле материала, предложенная модель хорошо справляется с описанием отклика сегнетоэлектроупругого материала на циклическую электрическую нагрузку. Однако с ростом частоты, как видно из анализа экспериментальных данных, происходит изменение формы гистерезиса. При частоте $f=1 \mathrm{~Hz}$ при увеличении электрического поля сначала происходит рост электрического смещения, а затем наблюдается его уменьшение. Предложенная модель описывает достаточно грубо появление указанного максимума.

При амплитудах воздействия, меньших коэрцитивного, наблюдается снижение точности прогноза. Для более точного описания поведения сегнетоэлектроупругих материалов в этом случае рационально использование моделей с порогом (аналог вязкопластичных моделей).

\section{Заключение}

Проведена верификация предложенной феноменологической модели нелинейного связанного электромеханического поведения поликристаллической сегнетопьезокерамики, учитывающей чувствительность к скорости нагружения, при циклическом нагружении. Установлены границы достоверного применения предложенной модели сегнетоэлектроупругого материала в пространстве амплитуд и частот нагружения. Хорошая точность прогнозов наблюдается при амплитудах внешнего электрического поля, превышающих коэрцитивное поле. Точность прогнозов модели падает с ростом частоты воздействия. Рассматриваемая модель непосредственно не учитывает доменный механизм переключения и ориентирована на использование при решении краевых задач с нетривиальной геометрией. В рамках единой модели оказалось возможным описание эффектов ползучести и чувствительности кривых гистерезиса к частоте внешнего воздействия. Сравнение результатов расчетов с прогнозами альтернативных моделей продемонстрировало более высокую точность предложенной модели при описании отклика материала при циклическом воздействии.

Исследование выполнено при поддержке гранта Российского научного фонда № 18-19-00413.

\section{Список литературы}

[1] Merz W.J. // Phys. Rev. 1954. Vol. 95. P. 690-704.

[2] Liu Q.D., Fleck N.A., Huber J.E., Chu D.P. // J. Europ. Ceramic Society. 2009. Vol. 29. P. 2289-2296.

[3] Zhou D., Kamlah M. // Acta Materialia. 2006. Vol. 54. P. 1389-1396.

[4] Heilig C., Haerdtl K.H. // IEEE Proceed. of the 6th IEKC, Montreux. 1998. P. 503-506.

[5] Guillon O., Thiebaud F., Delobelle P., Perreux D. // J. Europ. Ceramic Society. 2004. Vol. 24. P. 2547-2552.

[6] Fett T., Thun G. // J. Mater. Sci. Lett. 1998. Vol. 17. P. 1929 1931.

[7] Subbarao E.C., McQuarrie M.C., Buessem W.R. // 1957. Vol. 28. P. 1194-1200.

[8] Liu Q.D., Huber J.E. // J. Europ. Ceramic Society. 2006. Vol. 26. P. 2799-2806.

[9] Esaklul K.A., Gerberich W.W., Koepke B.G. // J. American Ceramic Society. 1980. Vol. 63. P. 25-30.

[10] Гладкий В.В., Кириков В.А., Нехлюдов С.В., Иванова E.C. // ФТТ. 1997. Т. 39. P. 2046-2052.

[11] Zhou D., Kamlah M., Munz D. // Proceed. SPIE. 2001. Vol. 4333. P. 64-70.

[12] Viehland D., Chen Y.H. // J. Appl. Phys. 2000. Vol. 88. P. 6696-6707.

[13] Гинзбург В.Л. // ЖЭТФ. 1945. Т. 15. С.739-749.

[14] Devonshire A.F. // Philos. Mag. 1949. Vol. 40. P. 1040-1063.

[15] Ландау Л.Д., Халатников И.М. // ДАН СССР. 1954. Т. 96. C. 469.

[16] Колмогоров А.Н. // Изв. АН СССР. Сер. Мат. 1937. № 3. C. 355.

[17] Avrami M. // J. Chem. Phys. 1939. Vol. 7. P. 1003.

[18] Ishibashi Y., Takagi Y. // J. Phys. Soc. Jpn. 1971. Vol. 31. P. 506-510.

[19] Orihara H., Hashimoto S., Ishibashi Y.J. // Phys. Soc. Jpn. 1994. Vol. 63. P. 1031-1035.

[20] Кукушкин С.А., Захаров М.А. // ФТТ. 2002. Т. 44. Вып. 2. C. 332-339.

[21] Захаров А.Ю., Бичурин М.И., Yan Y., Priya S. // ЖТФ. 2014. Т. 84 Вып. 8. С. 54-58.

[22] Захаров А.Ю., Бичурин М.И. // ЖТФ. 2015. Т. 85. Вып. 12. C. 69-73. 
[23] Semenov A.S., Liskowsky A.C., Balke H. // Int. J. for Numerical Methods in Engineering. 2010. Vol. 81. N 10. P. $1298-1340$.

[24] Bassiouny E., Ghaleb A.F., Maugin G.A. // Int. J. Eng. Sci. 1988. Vol. 26. P. 1279-1295.

[25] Kamlah M. // Continuum Mechanics and Thermodynamics. 2001. Vol. 13. P. 219-268.

[26] Landis C.M. // J. Mech. Phys. Sol. 2002. Vol. 50. P. 127-152.

[27] McMeeking R.M., Landis C.M. // Int. J. Eng. Sci. 2002. Vol. 40. P.1553-1577.

[28] Semenov A., Lobanov S. // Appl. Mechanics and Materials. 2015. Vol. 725-726. P. 961-966.

[29] Halphen B., Nguyen Q.S. // Journ. de Mecanique. 1975. Vol. 14. P. 39-63.

[30] Belov A.Yu., Kreher W.S. // Ferroelectrics. 2009. Vol. 391. P. 12-21.

[31] Семенов А.С. Вычислительные методы в теории пластичности. СПб.: Изд-во СПбГПУ, 2008. 211 с.

[32] Zhou D. Experimental investigation of non-linear constitutive behavior of PZT ceramics. Thesis. Germany: Universitat Karlsruhe, 2003. 156 p.

[33] Zhou D., Kamlah M., Munz D. // Proc. SPIE. Int. Soc. Opt. Eng. 2001. Vol. 4333. P. 64-70. 